\title{
Author Index Volume 20 (2012)
}

The issue number is given in front of the page numbers.

Adaikkalavan, R., X. Xie and I. Ray, Multilevel secure data stream processing: Architecture and implementation

(5) $547-581$

Ahn, G.-J., J. Jin and M. Shehab, Policy-driven role-based access management for ad-hoc collaboration

Alcalde, B., see Sun, Y.

Aliasgari, M., see Blanton, M.

Alvim, M.S., M.E. Andrés and C. Palamidessi, Quantitative information flow in interactive systems

Andrés, M.E., see Alvim, M.S.

Armando, A. and G. Lowe, Preface

Armando, A. and S. Ranise, Scalable automated symbolic analysis of administrative role-based access control policies by SMT solving

$(2,3) 223-257$

(4) $437-459$

(2,3) 259-305

(1) $3-50$

(1) $3-50$

(1) $1-1$

(4) 309-352

Backes, M., see Myers, A.

Barthe, G., J. Cuellar, J. Lopez and A. Pretschner, Preface

Basu, S., see Stakhanova, N.

Becker, M.Y., Information flow in trust management systems

Bezzi, M., S. De Capitani di Vimercati, S. Foresti, G. Livraga, P. Samarati and R. Sassi, Modeling and preventing inferences from sensitive value distributions in data release

Bielova, N. and F. Massacci, Iterative enforcement by suppression: Towards practical enforcement theories

Biskup, J., Dynamic policy adaptation for inference control of queries to a propositional information system

Blanton, M. and M. Aliasgari, Secure outsourced computation of iris matching

Bowen, B.M., V.P. Kemerlis, P. Prabhu, A.D. Keromytis and S.J. Stolfo, A system for generating and injecting indistinguishable network decoys

(6) $635-636$

(4) $307-308$

$(2,3) 169-198$

(6) $677-708$

(4) $393-436$

(1) $51-79$

(5) $509-546$

(2,3) 259-305

$(2,3) 199-221$

(6) $637-676$

Ciriani, V., S. De Capitani di Vimercati, S. Foresti, G. Livraga and P. Samarati, An OBDD approach to enforce confidentiality and visibility constraints in data publishing

Costa, G., P. Degano and F. Martinelli, Modular plans for secure service composition

Cuellar, J., see Barthe, G.

De Capitani di Vimercati, S., see Bezzi, M.

5) $463-508$

(1) $81-117$

(4) $307-308$

(4) $393-436$ 
De Capitani di Vimercati, S., see Ciriani, V.

(5) $463-508$

Degano, P., see Costa, G.

(1) $81-117$

Foley, S.N., see Zhou, H.

$(2,3) 119-167$

Foresti, S., see Bezzi, M.

(4) $393-436$

Foresti, S., see Ciriani, V.

(5) $463-508$

Garg, D. and F. Pfenning, Stateful authorization logic - Proof theory and a case study

(4) $353-391$

Hong, Y., J. Vaidya and H. Lu, Secure and efficient distributed linear programming

(5) $583-634$

Jin, J., see Ahn, G.-J.

(2,3) 223-257

Kemerlis, V.P., see Bowen, B.M.

$(2,3)$ 199-221

Keromytis, A.D., see Bowen, B.M.

Küsters, R., T. Truderung and A. Vogt, A game-based definition of coercion resistance and its applications

$(2,3)$ 199-221

Li, Y., Guest Editor's Preface

Livraga, G., see Bezzi, M.

Livraga, G., see Ciriani, V.

Lopez, J., see Barthe, G.

Lowe, G., see Armando, A.

Lu, H., see Hong, Y.

Martinelli, F., see Costa, G.

Massacci, F., see Bielova, N.

Mauw, S., see Sun, Y.

Myers, A. and M. Backes, Preface

(6) $709-764$

(5) $461-462$

(4) $393-436$

(5) $463-508$

(4) $307-308$

(1) $1-1$

(5) $583-634$

(1) $81-117$

(1) $51-79$

(4) $437-459$

(6) 635-636

Palamidessi, C., see Alvim, M.S.

Pang, J., see Sun, Y.

Pfenning, F., see Garg, D.

Prabhu, P., see Bowen, B.M.

Pretschner, A., see Barthe, G.

(1) $3-50$

(4) $437-459$

(4) $353-391$

$(2,3) 199-221$

(4) 307-308

Ranise, S., see Armando, A.

(4) $309-352$

(5) $547-581$

Samarati, P., see Bezzi, M.

Samarati, P., see Ciriani, V.

Sassi, R., see Bezzi, M.

Shehab, M., see Ahn, G.-J.

(4) $393-436$

(5) $463-508$

(4) $393-436$

(2,3) 223-257

Stakhanova, N., C. Strasburg, S. Basu and J.S. Wong, Towards costsensitive assessment of intrusion response selection

$(2,3) 169-198$

Stolfo, S.J., see Bowen, B.M.

$(2,3)$ 199-221 
Strasburg, C., see Stakhanova, N.

Sun, Y., C. Zhang, J. Pang, B. Alcalde and S. Mauw, A trust-augmented voting scheme for collaborative privacy management

Truderung, T., see Küsters, R.

Vaidya, J., see Hong, Y.

Vogt, A., see Küsters, R.

Wong, J.S., see Stakhanova, N.

Xie, X., see Adaikkalavan, R.

Zhang, C., see Sun, Y.

Zhou, H. and S.N. Foley, Fast automatic security protocol generation
$(2,3) 169-198$

(4) $437-459$

(6) $709-764$

(5) $583-634$

(6) $709-764$

(2,3) $169-198$

(5) $547-581$

(4) 437-459

$(2,3) 119-167$ 